\title{
A Fuzzy Hierarchical Multiple Criteria Group Decision Support System - Decider - and its Applications
}

Jun Ma, Guangquan Zhang, Jie Lu

\begin{abstract}
Decider is a Fuzzy Hierarchical Multiple Criteria Group Decision Support System (FHMC-GDSS) designed for dealing with subjective, in particular linguistic, information and objective information simultaneously to support group decision making particularly on evaluation. In this chapter, the fuzzy aggregation decision model, functions and structure of Decider are introduced. The ideas to resolve decision and evaluation problems we have faced in the development and application of Decider are presented. Two real applications of the Decider system are briefly illustrated. Finally, we discuss our further research in this area.
\end{abstract}

\section{Introduction}

Decision making is complex. An appropriate decision is often made by a group person in terms of several evaluation criteria. On the one hand, the rapidly increasing amount of data (information) provides necessary decision support, but at the same time, brings difficulties to appropriate decision making due to the reduced quality. Except for this reason, a decision maker's personal experience and knowledge in related fields are restricted. Hence, individual decision making often deviates from

Jun Ma

Centre of QCIS, Faculty of Engineering and Information Technology, University of Technology, Sydney (UTS)

e-mail: junmeit.uts.edu. au

Guangquan Zhang

Centre of QCIS, Faculty of Engineering and Information Technology, University of Technology, Sydney (UTS)

e-mail: zhanggeit.uts.edu. au

Jie Lu

Centre of QCIS, Faculty of Engineering and Information Technology, University of Technology, Sydney (UTS)

e-mail: jielu@it.uts.edu.au 
the appropriate one. Group decision making can redeem this deviation to some extent. On the other hand, an appropriate decision should be a result after deliberately synthesizing many related aspects of a decision problem. A decision just focusing a single aspect of a problem is dangerous in real application. Multi-criteria decision making (MCDM) can reduce the danger through consideration of a set, usually conflicting, of criteria simultaneously.

Multi-criteria group decision-making (MCGDM), which combines MCDM and GDM methods, has been proved to be a very effective technique to increase the degree of overall satisfaction for the final decision across the group [7], and is particularly suitable in problems such as quality evaluation, policy selection, employee nomination, and designing assessment $[4,19]$. These problems have some common features. For example, evaluation criteria are often in a multiple-level hierarchy; evaluators are from different departments; assessments are expressed in various forms. Traditional decision support systems can efficiently help decision makers resolve some of those problems. However, because they are mainly data-centred, they have obvious limitation to deal with subjective data which is a primary representation of assessments from evaluators. Therefore, how to efficiently deal with subjective information becomes an crucial issue in developing a real application of an MCGDM decision support system [6].

In practice, subjective data is often expressed by natural or artificial language, such as linguistic terms. Since Fuzzy sets technique is proved in practices that it is a powerful tool to handle subjective information, that combining fuzzy sets technique with MCGDM technique and studying FMCGDM technique is necessary and possible. In our opinion, FMCGDM technique is an important basis for developing people-centred intelligent systems including decision support. Based on aforementioned analysis and our related work, we developed the Fuzzy Hierarchical Multiple Criteria Group Decision Support System (FHMC-GDSS), named Decider, as a platform to test developed FMCGDM process algorithms. We also successfully applied this system to resolve subjective information process problems in industry applications. In this chapter, we will briefly introduce the main modules and the main functions of this system, and two of its applications.

The remaining sections in this chapter are organized as follows: Section 2 gives a simple overview of related research works on MCGDM and FMCGDM techniques and, then, lists used concepts and notions in the following sections; In Section 3 we will introduce the structure of the Decider system and its functions and implementation; Next, Section 4 illustrates two applications of the Decider system and gives a short analysis; Finally, we conclude the chapter and presents our further research.

\section{Related works}

Multi-criteria group decision making (MCGDM) is widely-used in various fields including managements [19], industry [20], social sciences [3], highway infrastructure management [23], spatial data processing [8], and urban water supply [10]. 
Techniques such as the Analytic Hierarchy Process (AHP) and evolutionary complutation have been applied in MCGDM [11, 22]. In practice, MCGDM is conducted in complicated context with heterogeneous information sources [18]. The collected information is often in two primary forms: subjective and objective. Effective process models and methods for integrating heterogeneous information are required.

Subjective information is often expressed by linguistic terms in real applications. Linguistic methods are typical techniques to integrate subjective information $[2,5,4]$. The core idea of existing linguistic methods is to develop an approximate aggregation operator to integrate linguistic information [16, 15, 24]. Because fuzzy set is the most used representation form of a linguistic term, most aggregation operators are established on fuzzy sets technique. Hundreds of aggregation operators have been developed and applied [1, 17]. However, existing linguistic methods only focus on linguistic terms process and pay little attention on objective information. In real applications, objective information is often some accurate measurements by means of devices and equipments with specific meanings and has special process requirements. Hence, it is necessary to establish information aggregation for subjective and objective information simultaneously.

In 2007, a fuzzy MCGDM decision algorithm was developed and implemented in an FMCGDM system [12] in our lab. Since then, some applications have been developed during collaboration with other researchers. Real applications indicated the great interested in such a decision support system and also presented more concrete and essential requirements. Based on the applications and their feedbacks, an expansion of that FMCGDM system is designed and named Decider, which is used as a testing and analysis platform of MCGDM algorithms and models. Since 2008, this system have been partly implemented and some planned functions of it have been readjusted based on requirements in applications. Section 3 will give more details of the Decider system.

Before introducing the Decider system, we give some basic definitions about fuzzy numbers and fuzzy algorithms which will be used in the following sections.

Definition 1 (Fuzzy set). A fuzzy set $\tilde{A}$ in a universe of discourse $X$ is characterized by a membership function $\mu_{\tilde{A}}(x)$ which associates with each example $x$ in $X$ a real number in the real interval $[0,1]$.

The function value $\mu_{\tilde{A}}(x)$ is called the membership degree of $x$ belonging to $\tilde{A}$.

Definition 2 (Cut set). The $\lambda$-cut set of a fuzzy set $\tilde{A}$ is defined by

$$
\tilde{A}_{\lambda}=\left\{x \in X \mid \mu_{\tilde{A}}(x) \geqslant \lambda\right\}
$$

where $\lambda \in[0,1]$ is a real number.

If $\tilde{A}_{\lambda}$ is a non-empty bounded closed interval in $X$, then it can be denoted by $\tilde{A}_{\lambda}=\left[\tilde{A}_{\lambda}^{L}, \tilde{A}_{\lambda}^{R}\right]$, where $\tilde{A}_{\lambda}^{L}$ and $\tilde{A}_{\lambda}^{R}$ are the lower and upper end points of the closed interval.

Definition 3 (Fuzzy number). [9] A fuzzy set $\tilde{a}$ on $\mathbb{R}$ is called a fuzzy number, if $\tilde{a}$ satisfies: 
(1) $\tilde{a}$ is a normal fuzzy set, i.e. $\tilde{a}_{1}$ is not empty;

(2) $\tilde{a}_{\lambda}$ is a closed interval for any $\lambda \in(0,1]$;

(3) the support of $\tilde{a}, \tilde{a}_{0+}$ is bounded.

In the following, the set of fuzzy numbers on $X$ is denoted by $\mathscr{F}(X)$.

Definition 4 (Basic Algorithms). For any $\tilde{a}, \tilde{b} \in \mathscr{F}\left(\mathbb{R}^{+}\right)$and $\alpha \in \mathbb{R}$, let

$$
\begin{aligned}
\tilde{a} \oplus \tilde{b} & =\bigcup_{\lambda \in(0,1]} \lambda\left[\tilde{a}_{\lambda}^{L}+\tilde{b}_{\lambda}^{L}, \tilde{a}_{\lambda}^{R}+\tilde{b}_{\lambda}^{R}\right], \\
\alpha \tilde{a} & =\bigcup_{\lambda \in(0,1]} \lambda\left[\alpha \tilde{a}_{\lambda}^{L}, \alpha \tilde{a}_{\lambda}^{R}\right], \\
\tilde{a} \otimes \tilde{b} & =\bigcup_{\lambda \in(0,1]} \lambda\left[\tilde{a}_{\lambda}^{L} \times \tilde{b}_{\lambda}^{L}, \tilde{a}_{\lambda}^{R} \times \tilde{b}_{\lambda}^{R}\right] .
\end{aligned}
$$

In Definition 4, we use $\oplus$ and $\otimes$ to replace + and $\times$ in conventional definitions in order to emphasize that the algorithm is applied to fuzzy numbers.

Definition 5 (Triangular fuzzy number). A triangular fuzzy number $\tilde{a}$ is defined by a triplet $\left(a_{0}^{L}, a, a_{0}^{R}\right)$ and the membership function $\mu_{\tilde{a}}(x)$ is given

$$
\mu_{\tilde{a}}(x)= \begin{cases}0, & x<a_{0}^{L} \\ \frac{x-a_{0}^{L}}{a-a_{0}^{L}}, & a_{0}^{L} \leqslant x<a \\ \frac{a_{0}^{R}-x}{a_{0}^{R}-a}, & a \leqslant x \leqslant a_{0}^{R} \\ 0, & a_{0}^{R}<x\end{cases}
$$

where $a=a_{1}^{R}=a_{1}^{L}$.

Definition 6 (Normalized positive fuzzy number). A fuzzy number $\tilde{a}$ is called a normalized positive fuzzy number if $0<a_{\lambda}^{L} \leqslant a_{\lambda}^{R} \leqslant 1$ for any $\lambda \in(0,1]$.

Definition 7 (Quasi-distance). Let $\tilde{a}, \tilde{b} \in \mathscr{F}(\mathbb{R})$ be two normalized positive fuzzy numbers, the quasi-distance of $\tilde{a}$ and $\tilde{b}$ is

$$
d(\tilde{a}, \tilde{b})=\left(\int_{0}^{1} \frac{1}{2}\left[\left(\tilde{a}_{\lambda}^{L}-\tilde{b}_{\lambda}^{L}\right)^{2}+\left(\tilde{a}_{\lambda}^{R}-\tilde{b}_{\lambda}^{R}\right)^{2}\right] \mathrm{d} \lambda\right)^{1 / 2} .
$$

\section{Decider: A Fuzzy Hierarchical Multiple Criteria Decision Support System}

This section introduces the design and implementation of the Decider system. As an expansion of our previous FMCGDM system, Decider also implemented the 
fuzzy MCGDM algorithm in that system. Besides, it also implemented other functions. At the beginning of its redesign, Decider is a testing and analysis platform of different MCGDM algorithms and models. However, with the progress in collaborative application developments, new requirements were concerned and new functions were added to this system.

Decider has some features to meet the demands of real applications.

(1) Decider is a cross-platform system. In applications, we noticed that some processes are not conducted on Windows operating system. Hence, when we redesigned the Decider system, we selected the Java programming language as developing tool and developed Decider on both MS Windows and Linux operating systems.

(2) Decider extents the hierarchies for criteria and evaluators. In our previous FMCGDM system, the level of criteria is restricted due to the limitation of used data structure, and only one level of evaluators was permitted. Considering the application requirements, we extended the hierarchies of criteria and evaluators in tree-like structures. Moreover, we have designed an information source level in order to represent network structure in criteria.

(3) Decider deals with subjective and objective simultaneously. It uses fuzzy numbers to represent subjective information such as linguistic terms, and applies a fuzzfication algorithm to convert objective information to subjective information.

The main components of Decider are shown in Fig. 1. Decider includes four basic modules, i.e., problem input, decision (MCGDM) process, decision display, and analysis/comparison. Users set the impacts and relationships/organizations of criteria, evaluators, alternatives, and other decision related information through "problem input" module. This information is then sent to the "decision process" module to generate decision result. The decision result is shown to users through the "decision play" module. Further, users can adjust decision parameters and process models by means of "analysis/comparison" module to check the change. Information between users and the Decider system forms two process circles called the basic process and the analysis process respectively.

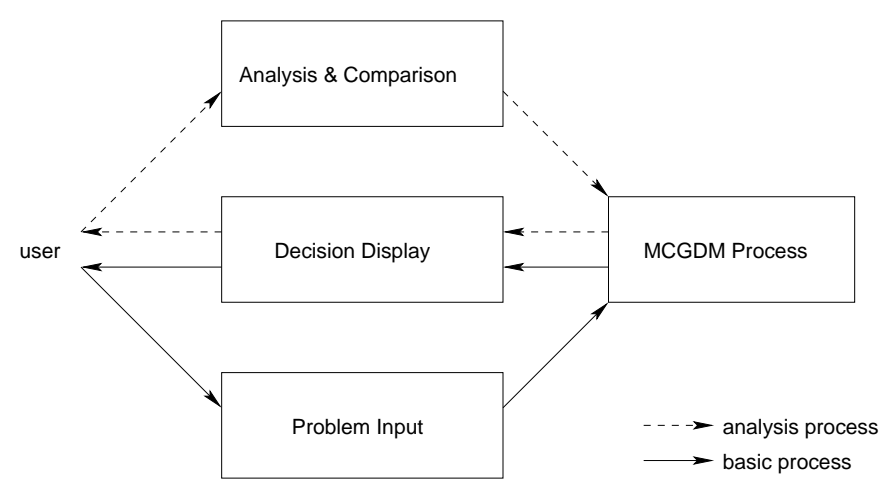

Fig. 1 Decider architecture 


\subsection{Decision Information Input}

In the "problem input" module, users mainly set two kinds of decision information. The first kind of decision information is called basic information which is directly collected for a decision problem. Basic information includes the relationships among criteria and their impacts (or weights, support degrees) to a decision problem; the organizations of evaluators and their impacts (or weights, reliabilities) to the decision problem; the assessments for each of alternatives (decision); and the information aggregation (fusion) strategy for the decision problem. The second kind of information is called conversion information which is taken from knowledge related to a decision problem. This information includes the relative distribution of assessments; the corresponding relations between subjective and objective assessments; and the cost/profit feature of criteria. The Decider system partially implements process for above information.

The most important basic information is the structure of criteria and their impacts. In the Decider system, the criteria related to a decision problem are organized in a multi-level hierarchy named criteria tree. The criteria tree is established through a cause-and-effect problem analysis from the general decision problem down to the detailed indicators. In the criteria tree, nodes except the root node are called criteria. In particular, leaf nodes of the criteria tree are called indicators; the children nodes of the root nodes are called aspects; and the rest criteria are called factors. The root node of the criteria tree represents the decision problem or decision goal/target derived from it; the aspects are general considerations which support the final decision; the indicators are detailed considerations on which assessments about alternatives are collected directly; and the factors illustrate the knowledge from indicators to final decision. Fig. 2 shows an example of a typical criteria tree in Decider.

Similar to the criteria tree, the Decider system uses an evaluator tree to represent organization of evaluators. There are two kinds of evaluators, i.e., the real evaluators and the virtual evaluators. A real evaluator refers to a person (expert) or a device, which provides assessments on alternatives directly. A virtual evaluator represents an evaluator group, a set of devices, or combination of human evaluators or devices. It is corresponding to a department or an assembly line in real applications.

Another kind of basic information is the impacts of criteria and evaluators on the decision problem and the assessments on alternatives. The Decider system provides two kinds of representations for above information, i.e., the subjective linguistic terms and the objective numeric values. Users can assign linguistic weights such as "Important" or numeric grade such as "4" to a criterion (or an evaluator) to describe its impact on the decision problem. Users can also input linguistic assessments such as "Very high" or numeric value such as "35.2" as assessment about an alternative. The used subjective and objective representations are listed in Table 1.

The conversion information is related to the processing method and derived from knowledge of the decision problem. Users can determine the corresponding relation between different information representation forms and select a process model from provided process models in the Decider system. As this information is closely related to decision process, details of it will be introduced in Section 3.2. 


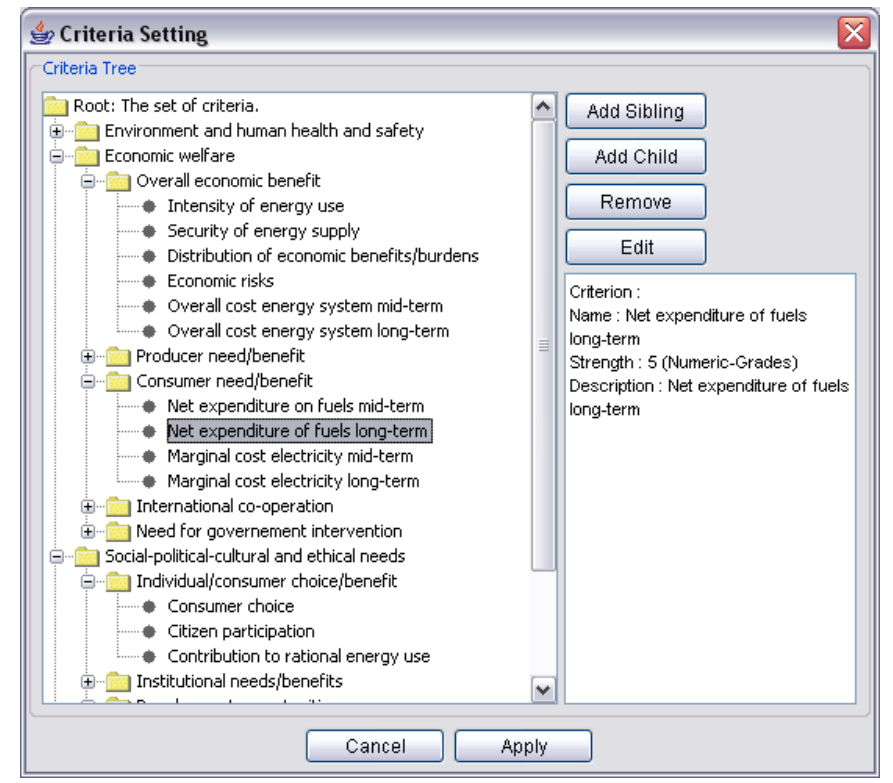

Fig. 2 An example of criteria tree.

Table 1 Information representation terms/values.

\begin{tabular}{|c|c|c|c|}
\hline Types & Named expression & Terms/values & Applied to \\
\hline \multirow[t]{4}{*}{ Subjective } & Standard Score (SS) & $0,1, \ldots, 100$ & $\begin{array}{l}\text { criteria } \\
\text { evaluators } \\
\text { assessments }\end{array}$ \\
\hline & Linguistic Weights (LW) & $\begin{array}{l}\text { Absolutely unimportant (AU) } \\
\text { Unimportant (U) } \\
\text { Less important (LI) } \\
\text { Important (I) } \\
\text { More important (MI) } \\
\text { Strongly important (SI) } \\
\text { Absolutely important (AI) }\end{array}$ & $\begin{array}{l}\text { criteria } \\
\text { evaluators }\end{array}$ \\
\hline & Linguistic Scores (LS) & $\begin{array}{l}\text { Lowest (LE) } \\
\text { Very low (VL) } \\
\text { Low (L), Medium (M) } \\
\text { High (H), Very high (VH) } \\
\text { highest (HE) }\end{array}$ & $\begin{array}{l}\text { assessments } \\
\text { criteria } \\
\text { evaluators }\end{array}$ \\
\hline & Numeric Grade (NG) & $1,2,3,4,5,6,7$ & $\begin{array}{l}\text { criteria } \\
\text { evaluators } \\
\text { assessments }\end{array}$ \\
\hline Objective & Range (R) & User defined interval of real numbers & assessments \\
\hline Boolean & & True (T), False (F) & assessments \\
\hline
\end{tabular}




\subsection{Decision Process}

Based on user selected process model and information about criteria, evaluators, and assessments, a basic process circle can be implemented. The main decision procedure is conducted in the "decision process" module. Decision process mainly implements the information conversion and the selected process model.

\subsubsection{Information conversion}

Information conversion conducts two kinds of information transformation. First, it determines the corresponding between different kinds of information representation forms. Second, it determines relative distributions of those terms or values. Concretely, suppose $S$ and $T$ are two sets of terms/values used in a decision process. The first transformation determines mapping between $S$ and $T$. Hence, for any $s_{i} \in S$, a term $t_{j}$ in $T$ is given as its corresponding and vice versa. Considering two representation forms may have different number of terms/values, a common reference is used in Decider. This common reference is the real interval [0, 1]. All six information representation forms in Table 1 are mapped into this interval. The second transformation determines the relative distribution of terms in a specific representation form. For instance, "Linguistic Weights (LW)" is composed of seven terms. Adjusting the mapping from LW to the interval [0,1], the images of the seven terms determine the relative relationship among them. The two transformations are supported in the current Decider system. However, for conversion consistency purpose, i.e., one transformation does not been changed by the other, the Decider system takes different strategies to convert subjective and objective information.

For subjective representations. A natural order is defined on SS, LW, LS, and NG for subjective terms/values such that

$$
\begin{array}{cc}
S S: & 0<1<2<\cdots<100 \\
L W: & A U<U<L I<I<M I<S I<A I \\
L S: & L E<V L<L<M<H<V H<H E \\
N G: & 1<2<3<4<5<6<7 .
\end{array}
$$

Then we map SS to $[0,1]$ such that

$$
x \mapsto x / 100, \quad x \in S S
$$

and map LS, LW, NG to SS based on "equal distance" or "equal ratio" settings and some initial assignments. Take LW for example. Suppose user assigns 0 to AU, and 90 to AI, and expects the rest terms to be placed by "equal distance". Then the terms U, LI, I, MI, and SI will be assigned 15, 30, 45, 60, and 75 respectively. If the user wants the rest terms to be placed by "equal ratio", and expects the ratio $\gamma$ is 3.0. Then based on this requirement and the system defined distribution function $s_{j+1}-s_{j}=\gamma \cdot\left(s_{j}-s_{j-1}\right)$, where $s_{j-1}, s_{j}$, and $s_{j+1}$ are sequential terms in LW, the 
terms U, LI, I, MI, and SI will be given 0, 1, 3, 9, and 27 respectively. If the user adds one more assignment 60 to I, then the terms U, LI, MI, and SI will take 20, 40, 70 , and 80 under the "equal distiance" setting and 5, 18, 62, and 69 under the "equal ratio" setting with ratio $\gamma=3.0$.

For objective representation. The Decider system converts directly the value to a fuzzy set on the real interval $[0,100]$. Decider requires users to determine three parameters to convert an objective value, i.e., the lower bound $(a)$, the upper bound $(b)$, and the preferred value $(p)$. Users also need to determine the interpretation of the preferred value from three interpretations: threshold value $(\mathrm{T})$, medium value $(\mathrm{M})$, and expected value (E). On the interpretation of the preferred value, Decider defines four kinds of orders for labeling an objective representation:

(1) the larger the better $(\mathrm{O} 1)$;

(2) the smaller the better $(\mathrm{O} 2)$;

(3) the near the better $(\mathrm{O} 3)$;

(4) the farther the better $(\mathrm{O} 4)$.

Therefore, an objective representation can be converted to a fuzzy set based on the combination of labelling order and interpretation of the preferred value. A linear transformation method is introduced in [14]. For instance, if the labelling order is $\mathrm{O} 3$ and the preferred value $p$ is interpreted as expected value (E), then Decider assigns 100 to $p$ and

$$
x \mapsto \begin{cases}\left\lfloor\frac{x-a}{p-a} \cdot 100\right\rfloor, & x \in[a, p] \\ \left\lfloor\frac{b-x}{b-p} \cdot 100\right\rfloor, & x \in[p, b] .\end{cases}
$$

For Boolean values, Decider will assign 100 to the value "True" and 0 to "False" by default. Under order reverse requirement, Decider will exchange the assignment of "True" and "False".

By the information conversion, information in different representation forms is mapped to SS. Next terms in SS will be mapped to normal positive fuzzy numbers on the interval $[0,1]$ in one of provided forms (triangular, quadratic, and exponential forms). For example, suppose $x$ is the image of term $t$ in SS, then the triangular form is given by

$$
\mu_{x}(y)= \begin{cases}\frac{y-x}{a}, & y \in[x-a, x], \\ \frac{y-x}{b}, & y \in[x, x+b],\end{cases}
$$

where $a, b$ are two parameters to be determined by users.

\subsubsection{Fuzzy information aggregation}

The core problem in the "decision process" module is the implementation of MCGDM process models and algorithms. Developing an aggregation method is the 
core technique in most MCGDM process models and algorithms. The Decider system implements a fuzzy aggregation algorithm as shown below. The fuzzy aggregation algorithm is applied to the criteria tree and the evaluator tree at the same time.

Suppose $c$ is a criterion in the criteria tree, $\tilde{v}_{1}, \tilde{v}_{2}, \tilde{v}_{n}$ are the assessments on its children nodes $c_{1}, c_{2}, \ldots, c_{n}$, and $w c_{1}, w c_{2}, \ldots, w c_{n}$ are the impacts of its children nodes to $c$. Here, $\tilde{v}_{j}$ and $w c_{j}(j=1,2, \ldots, n)$ are normalized positive fuzzy numbers on the real interval $[0,1]$. Then the assessment $\tilde{v}$ on $c$ is obtained by

$$
\tilde{v}=\sum_{j=1}^{n} \widetilde{w c_{j}} \otimes \tilde{v}_{j}
$$

where $\widetilde{w c_{j}}$ is the normalized impact for $w c_{j}$ given by

$$
\widetilde{w c}_{j}=\frac{w c_{j}}{\sum_{j}^{n}\left(w c_{j}\right)_{0}^{R}}, \quad j=1, \ldots, n,
$$

where $\left(w c_{j}\right)_{0}^{R}$ is the right-end point of the 0 -cut of $w c_{j}$.

Suppose $g$ is a virtual evaluator in the evaluator tree and $g_{1}, g_{2}, \ldots, g_{m}$ are its group members. Let $\tilde{u}_{1}, \tilde{u}_{2}, \ldots, \tilde{u}_{m}$ be the assessments from the $m$ group members and $w e_{1}, w e_{2}, \ldots, w e_{m}$ be their impacts on the group assessment of $g$. Therefore, the group assessment $\tilde{u}$ is obtained by

$$
\tilde{u}=\sum_{i=1}^{m} \widetilde{w e_{i}} \otimes \tilde{u}_{i}
$$

where $\widetilde{w e_{i}}$ is the normalized impact for $w e_{i}$ given by

$$
\widetilde{w e}_{i}=\frac{w e_{i}}{\sum_{i}^{m}\left(w e_{i}\right)_{0}^{R}}, \quad i=1, \ldots, m,
$$

where $\left(w e_{i}\right)_{0}^{R}$ is the right-end point of the 0 -cut of $w e_{i}$.

Notice that Eq. (11) and Eq. (13) is the same form, the order of aggregation on the criteria tree and the evaluator tree is not affecting. Hence, we can exchange the aggregation order.

The obtained assessment on the root node of the criteria tree and the evaluator tree will be used to generate the final decision. Suppose $\tilde{v}^{(i)}$ is the final assessment on the root node of the critera tree and the evaluator tree for the alternative $a_{i}, i=$ $1,2, \ldots, k$. To generate the decision, the Decider system will compare the assessment $\tilde{v}^{(i)}$ with two predefined ideal assessments $\tilde{v}^{(-)}$and $\tilde{v}^{(+)}$representing the worst and the best situations. $\tilde{v}^{(-)}$and $\tilde{v}^{(+)}$are two special normalized positive fuzzy numbers on the real interval $[0,1]$ defined as follows: 


$$
\tilde{v}^{(-)}=\left\{\begin{array}{ll}
1, & x=0 \\
0, & x \neq 0
\end{array}, \quad \tilde{v}^{(+)}=\left\{\begin{array}{ll}
1, & x=1 \\
0, & x \neq 1
\end{array} .\right.\right.
$$

The comparison result of the assessment $\tilde{v}^{(i)}$ and these two ideal assessments is a reference distance $d_{i}$ defined by

$$
d_{i}=\frac{1}{2}\left(d\left(\tilde{v}^{(i)}, \tilde{v}^{(-)}\right)+\left(1-d\left(\tilde{v}^{(i)}, \tilde{v}^{(+)}\right)\right)\right)
$$

where $d(\tilde{a}, \tilde{b})$ is a quasi fuzzy distance defined in Definition 7 . The reference distance $d_{i}$ is the final standard of genreating the decision. In most applications, for example profit related decisions, the decision is generated by the principle "the bigger the better". While in some applications, for instance cost related decisions, a decision is generated based on the reversion of above principle.

\subsection{Decision output}

Users can observe the generated result through the "decision display" module. This module displays the obtained reference distances of all alternatives in colour bars. Fig. 3 displays a snapshot of an example output. In this snapshot, area 1 displays the criteria tree; area 2 displays the evaluator tree; and area 3 displays the reference distance value of each alternative. The alternative with the biggest reference distance value is displayed in red. In real decision problem, this alternative is the best choice in general. Through graphic display, users can visually observe and compare these results.

By default, Decider will display the final results based on the root combination of the criteria tree and the evaluator tree. Considering users' different interests in a specific criterion or group, Decider can display the results accordingly. For instance, users can observe the results of all evaluators' synthesized assessment on specific criteria by selecting that criterion (from area $1 \mathrm{in} \mathrm{Fig.} \mathrm{3)} \mathrm{and} \mathrm{the} \mathrm{root} \mathrm{of} \mathrm{the} \mathrm{evaluator}$ tree (from area 2 in Fig. 3); users can observe a group's synthesized assessment on the whole problem by selecting that group (a virtual evaluator) and the root of the criteria tree. Users, therefore, can generate a whole picture of the solution to the problem.

\subsection{Analysis and comparison}

The procedure from users setting a decision problem, selecting processing model, to observing the decision results forms the first information process circle (the basic circle). The basic circle provides users with a general observation of the solution to the problem. If users need more observations, Decider provides the "analysis 


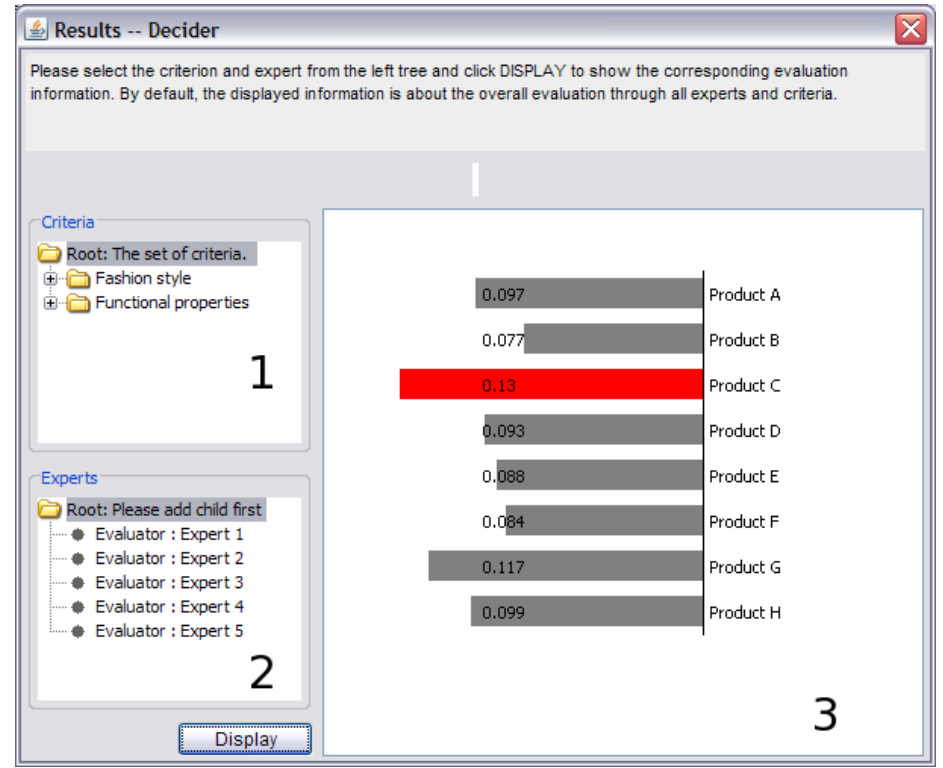

Fig. 3 Example of decision output

and comparison" module to help for it. This module is schemed but has not been completely implemented yet.

In the scheme, this module is going to implement three functions. The first one is sensitivity analysis. Decider will analyse the result change after adjusting the impacts of criteria and evaluators, and the assessments. The second one is decision models comparison. Decider will compare the result change after altering the aggregation operator and the process strategy of a used model and selecting different models. The third one is information representation comparison. Decider will compare the result change after changing the information representation forms.

Decider currently implements most of above functions in a limited way. For instance, it provides different decision models which have different requirements on information representation, aggregation operator, and decision strategy. Users can repeat the basic circle to conduct the listed analysis and comparison.

\section{Examples of applications}

This section will introduce three applications we have conducted using the Decider system. Detailed case studies can be found in the references [13, 21]. Here, we just focus on the particular requirement in those applications. 


\subsection{Long-term Scenarios of Belgian Energy Policy}

Long-term sustainable development is an issue of common concern in the whole world from developing countries to development countries. It involves problems in diversified forms. These problems include such as public health and security, environment protection and climate changes, as well as energy managements strategy. Formulating a long-term sustainable development policy is a typical multi-criteria group decision problem [19]. Since 2007, we have collaborated with researchers from Belgian Nuclear Research Centre to develop relevant knowledge and techniques for designing, evaluating, and selecting long-term energy strategy. This section gives a brief illustration of applying the Decider system to this problem.

The Long-term Belgian Energy Policy Evaluation (LBEPE) problem involves 63 criteria in 4 aspects, 16 factors and 43 indicators. Table 2 lists the relationship of the aspects, factors, and indicators. Based on Table 2, eight scenarios as alternatives are determined and named "MLCS," "MPCS," "MPLCS," "MPLCSI," "RLCS," "RPCS," "RPLCS," and "RPLCSI." 1

According to these criteria, we collected evaluation data from 10 experts. The collected data has three features. Due to the complexity of the problem, experts' views are expressed in linguistic terms in the evaluation procedure. Due to the lack of relevant knowledge, some views are not presented. Due to the criteria nature, some views using same term are with opposite meanings.

After necessary data pre-processing, experiments applying the Decider system to this problem are conducted. The evaluation results from the expert group and an individual are shown in Fig. 4 and Fig. 5. The values displayed on bars in Fig. 4 and Fig. 5 are relevant reference distances of those alternative. The bigger the value is, the bigger the corresponding reference distance is. From these figures, the difference and similarity between group and individual evaluations is easy to observe. For instance, from the group's viewpoint, the scenario "RPCS" is the best one; while expert e1 takes the scenario "RPLCS" as the best one. At the same time, they both think that the scenario "MLCS" is the worst.

\subsection{Well-being New Product Development}

We have also applied the Decider system to the well-being design theme evaluation of garment new product development in an establishing digital ecosystem. Digital ecosystem was presented in the FP6 framework programme and is continued as a research hotspot in the FP7 framework programme. Under its definition, the

\footnotetext{
${ }^{1}$ For each scenario, the initial letter "M" and "R" represent the Market world and the Rational Perspective world; and the following letters indicate possible energy strategies: $\mathrm{P}$ - nuclear phase out; LCS - low carbon capture and storage; I - import of electricity. For example, the scenario "MPLCS" is read: using the Market assumptions and assuming a nuclear phase out, no import and low potential for carbon capture and storage. In such a scenario, investments on renewable and cogeneration is therefore necessary. Detailed explanation of these scenarios is referred to [21].
} 
Table 2 Criteria of the long-term Belgian energy policy evaluation

\begin{tabular}{|c|c|}
\hline \multicolumn{2}{|c|}{ Environment \& human health and safety } \\
\hline Air pollution & $\begin{array}{l}\text { - Impacts of air pollution on human health: mid-term } \\
\text { - Impacts of air pollution on human health: long-term }\end{array}$ \\
\hline Occupational health & - Impacts on occupational health (gas + coal) \\
\hline Radiological health impacts & $\begin{array}{l}\text { - Radiological health impacts (nuclear) } \\
\text { - Need for long-term management of HLW }\end{array}$ \\
\hline Aesthetic impacts & $\begin{array}{l}\text { - Visual impact on landscape } \\
\text { - Noise amenity }\end{array}$ \\
\hline Other environmental impacts & $\begin{array}{l}\text { - Impact on natural ecosystem (air pollution): mid-term } \\
\text { - Impact on natural esosystem (air pollution): long-term } \\
\text { - Environmental impact from solid waste (coal) }\end{array}$ \\
\hline Resource use & $\begin{array}{l}\text { - Land use } \\
\text { - Water use }\end{array}$ \\
\hline Other energy related pressures & $\begin{array}{l}\text { - Catastrophic risk: nuclear } \\
\text { - Geographical distribution risk/benefits }\end{array}$ \\
\hline \multicolumn{2}{|l|}{ Economic welfare } \\
\hline Overall economic benefit & $\begin{array}{l}\text { - Intensity of energy use } \\
\text { - Security of energy supply } \\
\text { - Distribution of economic benefits / burdens } \\
\text { - Ecosuppressnomic risks } \\
\text { - Overall cost energy system: } 2010 \\
\text { - Overall cost energy system: } 2030\end{array}$ \\
\hline Producer need/benefit & $\begin{array}{l}\text { - Overall cost energy system: } 2050 \\
\text { - Ability to provide specialist market } \\
\text { - Marginal cost electricity: mid-term }\end{array}$ \\
\hline Consumer need/benefit & - Marginal cost electricity: long-term \\
\hline International cooperation & $\begin{array}{l}\text { - Strategic factors for export } \\
\text { - Compatibility with international R\&D agenda }\end{array}$ \\
\hline Need for government intervention & - Amount of direct or indirect subsidies needed \\
\hline \multicolumn{2}{|c|}{ Social, political, cultural and ethical needs } \\
\hline Individual/consumer choice/benefit & $\begin{array}{l}\text { - Consumer choice } \\
\text { - Citizen participation } \\
\text { - Contribution to rational energy use }\end{array}$ \\
\hline Institutional needs & $\begin{array}{l}\text { - Degree of decentralisation } \\
\text { - Need for intermediary storage of spent fuel } \\
\text { - Control and concentration of power } \\
\text { - Influence on political decision-making } \\
\text { - Need for socio-political stability } \\
\text { - Need for direct political intervention } \\
\text { - Reversibility of technology choice } \\
\text { - Knowledge specialisation } \\
\text { - Need for institutional non-proliferation measures }\end{array}$ \\
\hline Development opportunities & $\begin{array}{l}\text { - Potential for technology transfer } \\
\text { - Leaving resources for development } \\
\text { - Equity (general) }\end{array}$ \\
\hline Jobs & - Job opportunities \\
\hline
\end{tabular}




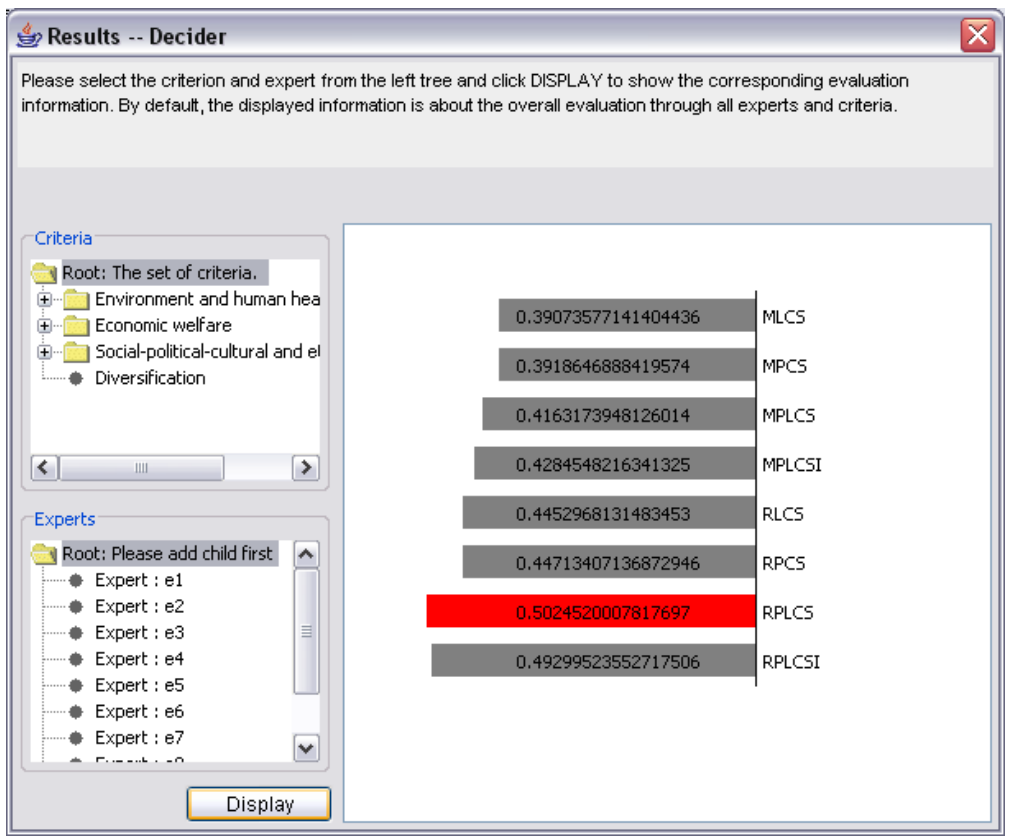

Fig. 4 Evaluation result from the expert group.

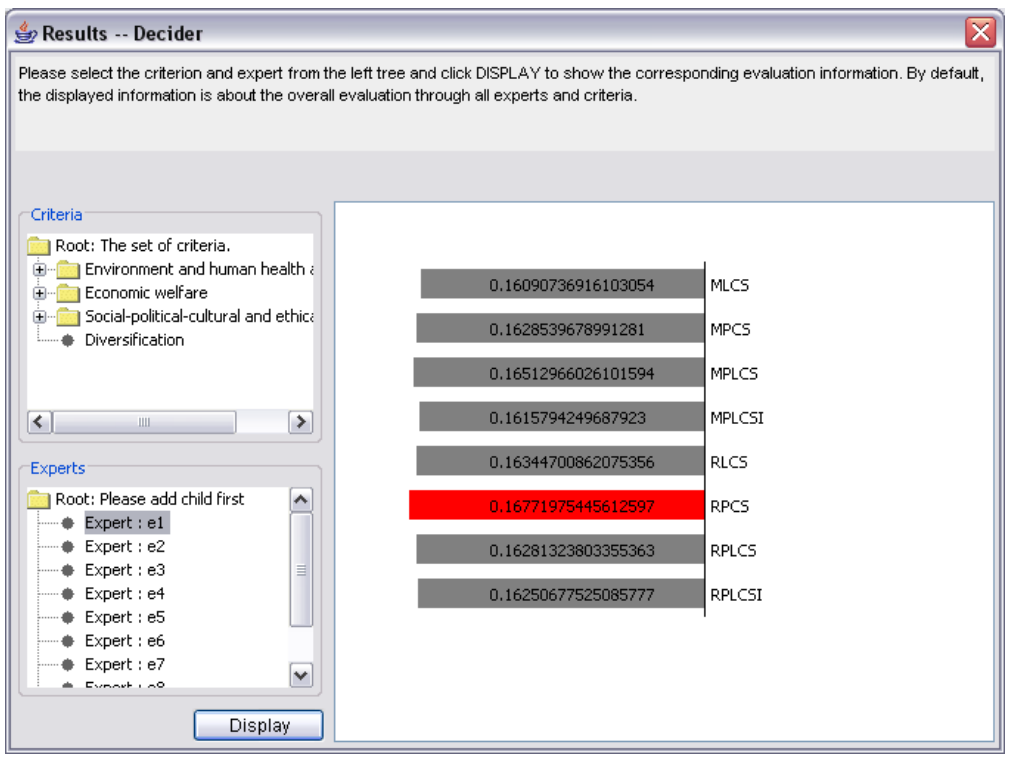

Fig. 5 Evaluation result from the expert 1. 
user-centred product and service development is a primary research activity for garment enterprises in their product development procedure. These enterprises need to evaluate their product design before launch a new product to a competitive market. This evaluation procedure must combine human actions and cognition with their manufacturing.

Well-being is a design theme with both psychological and sociological features. That evaluating a new product design is appropriate or inappropriate for the wellbeing theme involves not only subjective assessments from customers and experts but also objective measurements in the manufacturing procedure simultaneously. The criteria tree is given in Table 3. Observing the criteria tree, we know that some criteria are with objective information form. Data for these criteria is generally not provided by human beings but by specific devices.

Based on the criteria, a survey was conducted among garment experts and customers. We collected assessments from these people on all subjective indicators and measurements from devices on all objective indicators. Considering that all specific devices from which the objective data is collected coexist in a whole manufacturing procedure and affect a product together, we set a virtual evaluator to represent all specific devices. Then, applying the Decider system, we evaluated eight product designs. The evaluation results are shown in Fig. 6 - Fig. 8. Comparing these results, we can observe that the product $\mathrm{C}$ is the one which is appropriate for the well-being theme. Because both human evaluators and the devices reach the same assessment, the manufacturing setting for product $\mathrm{C}$ need not to be adjusted. However, for the products $\mathrm{B}$ and $\mathrm{D}$, the human evaluators and the devices give different assessments. Hence, the manufacturing settings for them should be adjusted.

\subsection{Further Discussion}

Practices of applying the Decider system to many real applications indicate that developing such a decision support system is necessary and urgent. Different from traditional decision support system, Decider can partly handle subjective information. We have noticed during developing above applications that one of important factor which affects appropriate decision is the subjective information process. This is because that human is the centre of a decision problem and communication is a main channel of subjective information. Hence, establishing people-centred decision support system is an efficient way to improve decision quality. That is a main reason for us developing such a system.

Another important point we found in those applications is that establishing the connection between objective and subjective information is an implied way for transferring knowledge and perception. In manufacturing, experts' and customers' knowledge and perception cannot be used directly. Manufacturing is measured by devices, i.e. by objective data; while knowledge and perception is expressed by subjective information. To produce products satisfy customers' perception, transferring those perception to objective data is necessary and crucial. 
Table 3 Criteria tree of Well-being design theme evaluation problem.

\begin{tabular}{|c|c|}
\hline Criteria name & Information form \\
\hline Fashion stype & Subjective (S) \\
\hline Protection & $\mathrm{S}$ \\
\hline Health & $\mathrm{S}$ \\
\hline Pleasure & $\mathrm{S}$ \\
\hline Serenity & $\mathrm{S}$ \\
\hline Relaxation & $\mathrm{S}$ \\
\hline Cocooning & $\mathrm{S}$ \\
\hline Warmth & $\mathrm{S}$ \\
\hline Health & $\mathrm{S}$ \\
\hline Sport & $\mathrm{S}$ \\
\hline Serenity & $\mathrm{S}$ \\
\hline Pleasure & $\mathrm{S}$ \\
\hline Holiday & $\mathrm{S}$ \\
\hline Relaxation & $\mathrm{S}$ \\
\hline Cocooning & $\mathrm{S}$ \\
\hline Dynamism & $\mathrm{S}$ \\
\hline Sport & $\mathrm{S}$ \\
\hline Pleasure & $\mathrm{S}$ \\
\hline Relaxation & $\mathrm{S}$ \\
\hline Coolness & $\mathrm{S}$ \\
\hline Health & $S$ \\
\hline Pleasure & S \\
\hline Holiday & S \\
\hline Relaxation & S \\
\hline Functional properties & $\mathrm{S}$ \\
\hline Fabric handle & $\mathrm{S}$ \\
\hline Extensibility & Objective (O) \\
\hline Density & $\mathrm{O}$ \\
\hline Compressibility & $\mathrm{O}$ \\
\hline Flexibility & $\mathrm{O}$ \\
\hline Surface friction & $\mathrm{O}$ \\
\hline Resilience & $\mathrm{O}$ \\
\hline Surface contour & $\mathrm{O}$ \\
\hline Thermal-Wet sensation & $\mathrm{O}$ \\
\hline Smell & $\mathrm{O}$ \\
\hline Sound & $\mathrm{O}$ \\
\hline Wash and care & $\mathrm{S}$ \\
\hline Wash requirement & $\mathrm{S}$ \\
\hline Iron requirement & $\mathrm{S}$ \\
\hline Storage requirement & $\mathrm{S}$ \\
\hline Durability & $\mathrm{O}$ \\
\hline
\end{tabular}




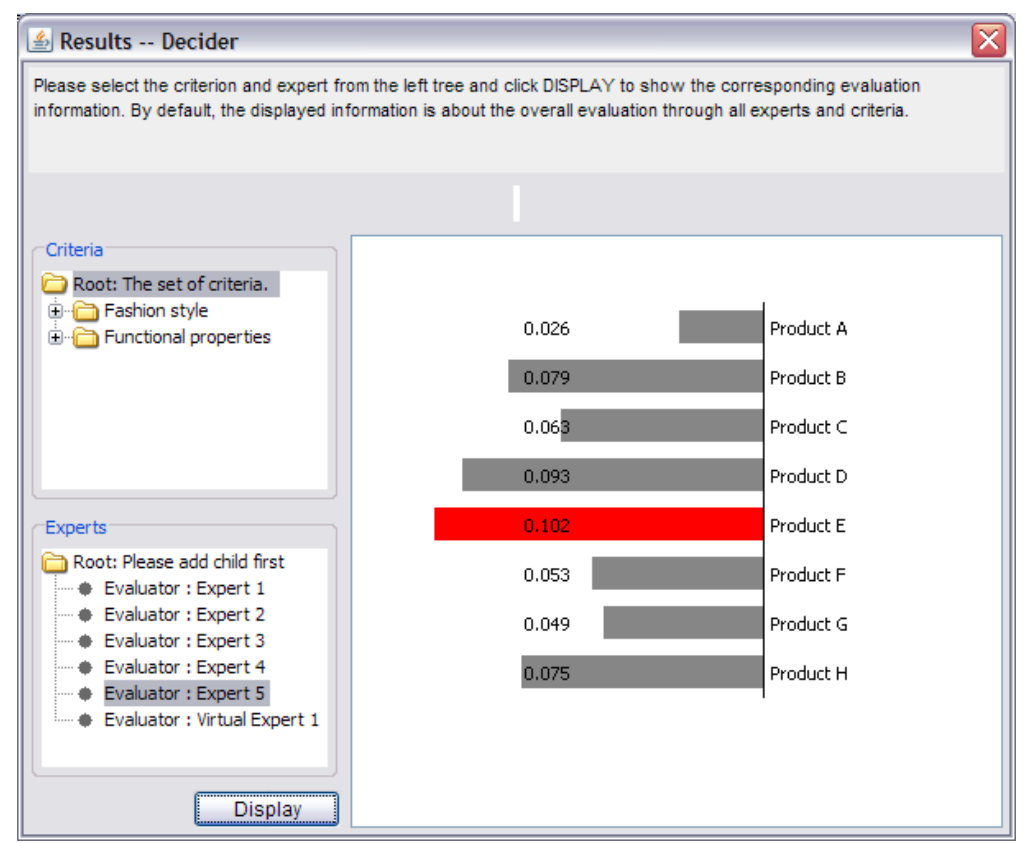

Fig. 6 Evaluation result on well-being design by an individual human evaluator.

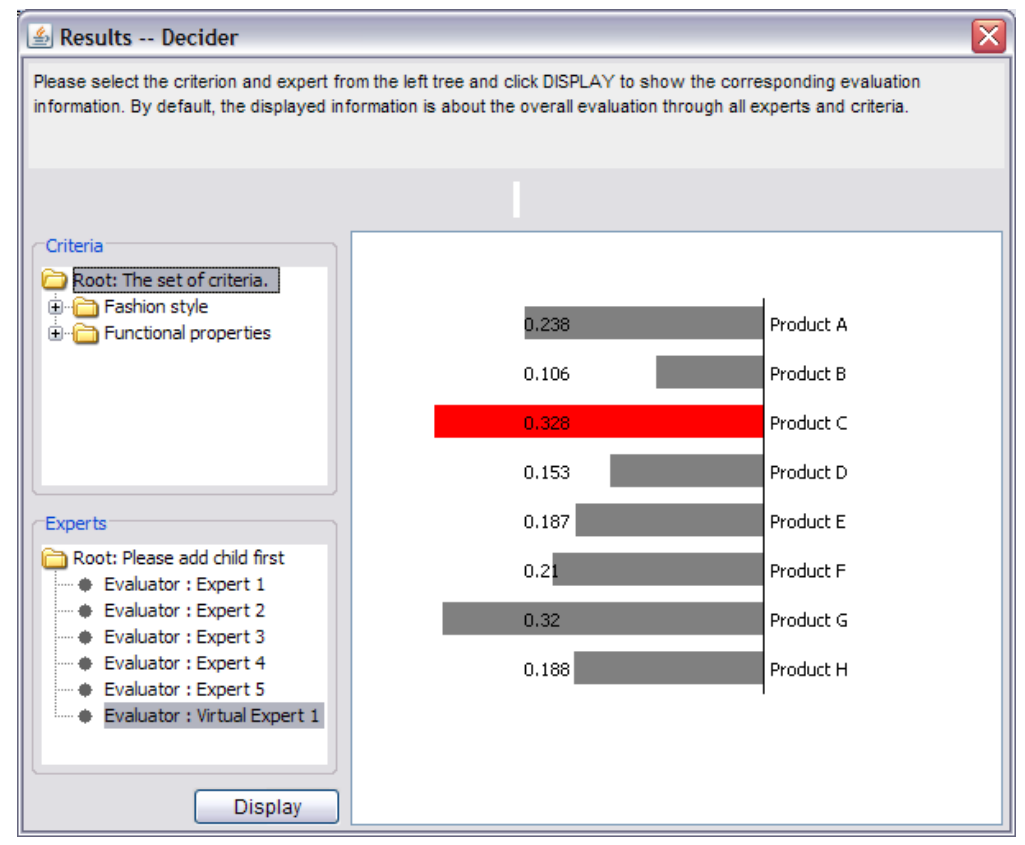

Fig. 7 Evaluation result on well-being design by devices. 


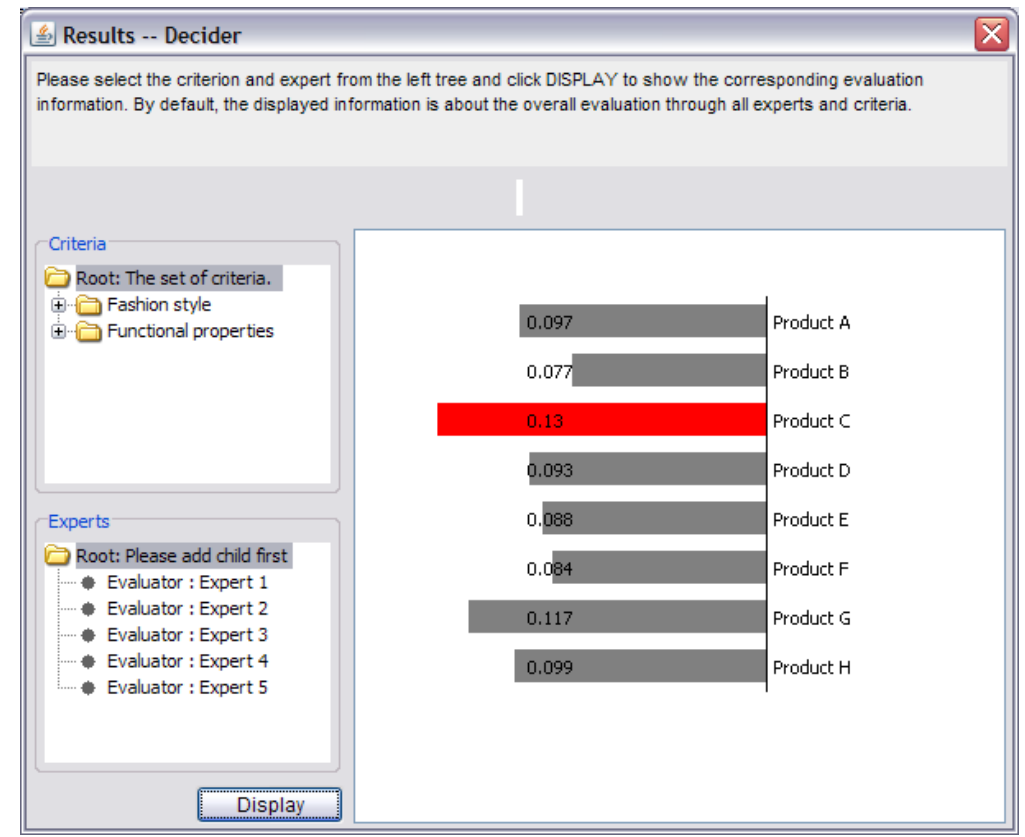

Fig. 8 Evaluation result on well-being design by all human evaluators.

Another starting point of our developing the Decider system is establishing a testing platform of decision algorithms and models. Since the requirements of real applications vary from one to another, it is possible to verify which algorithm or model is suitable to which situation and requirement. This work is very helpful for both theoretical research and industrial application. More work need to be done not only for improving the developed system but also for advancing related research.

\section{Conclusions}

In this chapter, we introduced a fuzzy hierarchical multiple criteria group decision support system - Decider. We overviewed the structure and function of the Decider system and its applications on two typical multiple criteria decision problems. Through developing real applications, we concluded that 1) the Decider system can partly resolve subjective information process in decision problems; but more work needs to be done to improve the developed system; 2) developing and deploying people-centred decision support systems is necessary in real application fields; 3) advancing research on human decision making with subjective information will help establish people-centred decision support. These issues are also our future works. 
Acknowledgements The work presented in this paper was supported by Australian Research Council (ARC) under Discovery Project DP0880739.

\section{References}

1. Tomasa Calvo, Gaspar Mayor, and Radko Mesiar. Aggregation Operators: New Trends and Applications. Number 97 in Studies in Fuzziness and Soft Computing. Physica-Verlag, Springer, Heidelberg, 2002.

2. M. Delgado, F. Herrera, E. Herrera-Viedma, J. L. Verdegay, and M. A. Vila. Aggregation of linguistic information based on a symbolic approach. In L.A. Zadeh and J. Kacpryzk, editors, Computing with Words in Information/Intelligent Systems I. Foundations, pages 428-440. Physica-Verlag, 1999.

3. Michel Grabisch and Christophe Labreuche. Fuzzy Measures and Integrals in MCDA. In José Figueira, Salvatore Greco, and Matthias Ehrgott, editors, Multiple Criteria Decision Analysis: State of the Art Surveys, volume 78 of International Series in Operations Research \& Management Science, chapter 14, pages 563-604. Springer, New York, 2005.

4. Francisco Herrera, E. Herrera-Viedma, and F. Chiclana. Multiperson decision-making based on multiplicative preference relations. European J. Operational Research, 129:372-385, 2001.

5. Francisco Herrera, E. Lopez, and M.A. Rodriguez. A linguistic decision model for promotion mix management solved with genetic algorithms. Fuzzy Sets and Systems, 131:47-61, 2002.

6. Enrique Herrera-Viedma, Francisco Chiclana, Francisco Herrera, and Sergio Alonso. Group decision-making model with incomplete fuzzy preference relations based on additive consistency. IEEE Transactions on Systems, Man, and Cybernetics - Part B: Cybernetics, 37(1):176-189, 2007.

7. Van-Nam Huynh and Yoshitern Nakamori. A satisfactory-oriented approach to multiexpert decision-making with linguistic assessments. IEEE Transactions on Systems, Man, and Cybernetics - Part B: Cybernetics, 35(2):184-196, 2005.

8. James C. Ascough II, Harriet D. Rector, Dana L. Hoag, Gregory S. McMaster, Bruce C. Vandenberg, Marvin J. Shaffer, Mark A. Weltz, and Lajpat R. Ahjua. Multicriteria spatial decision support systems: overview, applications, and future research directions. In Proceedings of Integrated Assessment and Decision Support (iEMSs 2002), pages 175-180, Lugano, Switzerland, June 2002.

9. Geogre J. Klir and Bo Yuan. Fuzzy Sets and Fuzzy Logic: Theory and Applications. PrenticeHall Inc., NJ, USA, 1995.

10. Prashanthi Nirmala Kodikara. Multi-Objective Optimal Operation of Urban Water Supply Systems. PhD thesis, School of Architectural, Civil and Mechanical Engineering, Feb. 2008.

11. Marco Laumann, Eckart Zitzler, and Lothar Thiele. Multiple criteria decision support by evolutionary computation. In L. M. Hilty and P.W. Gilgen, editors, Sustainability in the Information Society, 15th International Symposium Informatics for Environmental Protection, Zurich, October 2001. Marburg: Metropolis Verlag.

12. Jie Lu, Guangquan Zhang, Da Ruan, and Fengjie Wu. Multi-Objective Group Decision Making - Methods, Software and Applications with Fuzzy Set Technology. Imperial College Press, London, 2007.

13. Jie Lu, Yijun Zhu, Xianyi Zeng, Ludovic Koehl, Jun Ma, and Guangquan Zhang. A fuzzy decision support system for garment new product development. In AI08: Proceedings of the 21st Australasian Joint Conference on Artificial Intelligence, pages 532-543, Berlin, Heidelberg, 2008. Springer-Verlag.

14. Jun Ma, Jie Lu, and Guangquan Zhang. Decider: A fuzzy multi-criteria group decision support system. Knowledge-Based Systems, 23(1):23-31, 2010.

15. Jean-Luc Marichal. An axiomatic approach of the discrete Choquet integral as a tool to aggregate interacting criteria. IEEE Transactions on Fuzzy Systems, 8(6):800-807, 2000. 
16. Jean-Luc Marichal. An axiomatic approach of the discrete Sugeno integral as a tool to aggregate interacting criteria in a qualitative framework. IEEE Transactions on Fuzzy Systems, 9(1):164-172, 2001.

17. Radko Mesiar, Anna Kolesárová, Tomasa Calvo, and Magda Komorníková. A review of aggregation functions. In Humberto Bustince, Francisco Herrera, and Javier Montero, editors, Fuzzy Sets and Their Extensions: Representation, Aggregation and Models, volume 220 of Studies in Fuzziness and Soft Computing, pages 121-144. Springer Berlin/Heidelberg, 2008.

18. Amihai Motro and Philipp Anokhin. Fusionplex: resolution of data inconsistencies in the integration of heterogeneous information sources. Information Fusion, 7(2):176-196, 2006.

19. Giuseppe Munda. A conflict analysis approach for illuminating distributional issues in sustainability policy. European Journal of Operational Research, 194(1):307-322, 2009.

20. Da Ruan, Jun Liu, and Roland Carchon. Linguistic assessment approach for managing nuclear safeguards indicator information. Logistic Information Management, 16(6):401-419, 2003.

21. Da Ruan, Jie Lu, Erik Laes, Guangquan Zhang, and Jun Ma. Multi-criteria group decision support with linguistic variables in long-term scenarios for belgian energy policy. Journal of Universal Computer Science, 15(1):103-120, 2010.

22. Pulyamon Vihakapirom and Raymond Koon-Ying Li. A framework for distributed group multi-criteria decision support systems. In Proceedings of the Ninth Australia World Wide Web Conference, Hyatt Sanctuary Cove, Gold Coast, July 2003.

23. Jana Šelih, Anžej Kne, Aleksander Srdić, and Marjan Žura. Multiple-criteria decision support system in highway infrastructure management. Transport, 23(4):299-305, 2008.

24. Ronald R. Yager. Families of OWA operators. Fuzzy Sets and Systems, 59:125-148, 1993. 
Enrique Herrera-Viedma José Luis García-Lapresta Janusz Kacprzyk

Mario Fedrizzi

Hannu Nurmi Sławomir Zadrożny (Eds.)

\section{Consensual Processes}


Enrique Herrera-Viedma, José Luis García-Lapresta, Janusz Kacprzyk, Mario Fedrizzi, Hannu Nurmi, and Sławomir Zadrożny (Eds.)

Consensual Processes 


\section{Studies in Fuzziness and Soft Computing, Volume 267}

\section{Editor-in-Chief}

Prof. Janusz Kacprzyk

Systems Research Institute

Polish Academy of Sciences

ul. Newelska 6

01-447 Warsaw

Poland

E-mail: kacprzyk@ibspan.waw.pl

Further volumes of this series can be found on our homepage: springer.com

Vol. 253. Badredine Arfi

Linguistic Fuzzy Logic Methods in Social

Sciences, 2010

ISBN 978-3-642-13342-8

Vol. 254. Weldon A. Lodwick,

Janusz Kacprzyk (Eds.)

Fuzzy Optimization, 2010

ISBN 978-3-642-13934-5

Vol. 255. Zongmin Ma, Li Yan (Eds.)

Soft Computing in XML Data

Management, 2010

ISBN 978-3-642-14009-9

Vol. 256. Robert Jeansoulin, Odile Papini,

Henri Prade, and Steven Schockaert (Eds.)

Methods for Handling Imperfect

Spatial Information, 2010

ISBN 978-3-642-14754-8

Vol. 257. Salvatore Greco,

Ricardo Alberto Marques Pereira,

Massimo Squillante, Ronald R. Yager,

and Janusz Kacprzyk (Eds.)

Preferences and Decisions, 2010

ISBN 978-3-642-15975-6

Vol. 258. Jorge Casillas and

Francisco José Martínez López

Marketing Intelligent Systems

Using Soft Computing, 2010

ISBN 978-3-642-15605-2

Vol. 259. Alexander Gegov

Fuzzy Networks for Complex

Systems, 2010

ISBN 978-3-642-15599-4

Vol. 260. Jordi Recasens

Indistinguishability Operators, 2010

ISBN 978-3-642-16221-3
Vol. 261. Chris Cornelis, Glad Deschrijver, Mike Nachtegael, Steven Schockaert, and Yun Shi (Eds.)

35 Years of Fuzzy Set Theory, 2010

ISBN 978-3-642-16628-0

Vol. 262. Zsófia Lendek, Thierry Marie Guerra,

Robert Babuška, and Bart De Schutter

Stability Analysis and Nonlinear Observer

Design Using Takagi-Sugeno

Fuzzy Models, 2010

ISBN 978-3-642-16775-1

Vol. 263. Jiuping Xu and Xiaoyang Zhou Fuzzy-Like Multiple Objective Decision Making, 2010

ISBN 978-3-642-16894-9

Vol. 264. Hak-Keung Lam and

Frank Hung-Fat Leung

Stability Analysis of Fuzzy-Model-Based

Control Systems, 2011

ISBN 978-3-642-17843-6

Vol. 265. Ronald R. Yager, Janusz Kacprzyk, and Prof. Gleb Beliakov (Eds.)

Recent Developments in the Ordered Weighted Averaging Operators: Theory and Practice, 2011 ISBN 978-3-642-17909-9

Vol. 266. Edwin Lughofer

Evolving Fuzzy Systems - Methodologies, Advanced Concepts and Applications, 2011 ISBN 978-3-642-18086-6

Vol. 267. Enrique Herrera-Viedma,

José Luis García-Lapresta, Janusz Kacprzyk, Mario Fedrizzi, Hannu Nurmi, and Sławomir Zadrożny (Eds.) Consensual Processes, 2011 ISBN 978-3-642-20532-3 
Enrique Herrera-Viedma, José Luis García-Lapresta, Janusz Kacprzyk, Mario Fedrizzi, Hannu Nurmi, and Sławomir Zadrożny (Eds.)

\section{Consensual Processes}




\section{Editors}

Prof. Enrique Herrera-Viedma

Prof. Dr. Mario Fedrizzi

Universidad Granada

Depto. Ciencias de la

Computación e

Inteligencia Artificial

C/ Periodista Daniel Saucedo

Aranda s/n

18071 Granada, Spain

E-mail: viedma@decsai.ugr.es

José Luis García-Lapresta

Universidad de Valladolid

Departamento de Economía

Aplicada

Avenida Valle Esgueva 6

47011 Valladolid, Spain

E-mail: lapresta@eco.uva.es

Janusz Kacprzyk

Polish Academy of Sciences

Systems Research Institute

ul. Newelska 6

Università di Trento

Fac. Economia

Dipto. Informatica e Studi

Aziendali

Via Inama 538100 Trento Trento, Italy

E-mail: fedrizzi@cs.unitn.it

Hannu Nurmi

University of Turku

Department of Political Science

and

Contemporary History

Turku, Finland

E-mail: hnurmi@utu.fi

Sławomir Zadrożny

Polish Academy of Sciences

Systems Research Institute

ul. Newelska 6

01-447 Warsaw, Poland

E-mail: zadrozny@ibspan.waw.pl

01-447 Warsaw, Poland

E-mail: kacprzyk@ibspan.waw.pl

ISBN 978-3-642-20532-3

e-ISBN 978-3-642-20533-0

DOI 10.1007/978-3-642-20533-0

Studies in Fuzziness and Soft Computing

ISSN 1434-9922

Library of Congress Control Number: 2011927922

(c) 2011 Springer-Verlag Berlin Heidelberg

This work is subject to copyright. All rights are reserved, whether the whole or part of the material is concerned, specifically the rights of translation, reprinting, reuse of illustrations, recitation, broadcasting, reproduction on microfilm or in any other way, and storage in data banks. Duplication of this publication or parts thereof is permitted only under the provisions of the German Copyright Law of September 9, 1965, in its current version, and permission for use must always be obtained from Springer. Violations are liable to prosecution under the German Copyright Law.

The use of general descriptive names, registered names, trademarks, etc. in this publication does not imply, even in the absence of a specific statement, that such names are exempt from the relevant protective laws and regulations and therefore free for general use.

Typeset \& Cover Design: Scientific Publishing Services Pvt. Ltd., Chennai, India.

Printed on acid-free paper

987654321

springer.com 


\section{Preface}

Consensus has been a much talked about word for centuries, maybe millennia since people have always been aware of its importance for arriving at proper decisions which have had a long lasting impact on life of groups of people, countries or even civilizations. Needless to say that the growing complexity of the present world has made the word to be used so frequently nowadays.

The first question is, as always, the one that relates to the very meaning of the word. In general, one can say that by consensus is meant, on the one hand, a general agreement within the group of people, or agents, both human and software, in a more general setting. Clearly, in the strict meaning the general agreement has been viewed in a "yes-no" way, that is, in the sense of full and unanimous agreement. Since this may be an unreachable ideal, people has been for a long time trying to make this definition more realistic and have replaced this ideal concept by a more realistic one that encompasses all forms of partial, graded, etc. agreements within a group.

The second sense of this word is related to a process of reaching consensus which aims at reaching an agreement, possibly a high one, of all, or most, agents. It can involve the resolution and/or mitigation of some minor objections concerning options or aspects in question or individual agents involved.

It is easy to see that the second sense of consensus does involve the first sense because the process of reaching an agreement must be related to some assessment of what, and to which extent the current agreement within the group exists.

This volume is concerned with consensus reaching processes which may occur both within human groups and groups of intelligent agents. We adopt, first of all, a modern and realistic definition of consensus that considers consensus not necessarily as full and unanimous agreement but as agreement to some extent. This calls for some soft computational tools and techniques and we mainly use fuzzy and possibilistic ones to be able to account for imprecision in the very meaning of many concepts, issues and properties that play a role in both that more realistic definition of consensus and the very process of consensus reaching.

The volume is intended to provide a comprehensive coverage of various issues related to consensus and consensual processes.

Part I focuses on consensus from dynamical points of view. The chapters are concerned with fundamental issues of consensus dynamics:, notably aggregation, ranking, opinion changing propensity, preference modeling, etc. 
D. Eckert and Ch. Klamler ("Distance-Based Aggregation Theory") consider the problem of aggregating several objects into an object that best represents them which is a central problem in diverse fields exemplified by economics, sociology, political science, statistics, biology, etc. This problem[s] has also been extensively dealt with in the theory of social choice which analyses the aggregation of individual preferences into a collective preference. In this context, the idea of a consensus is normatively particularly appealing. A natural way to operationalize the consensus among a group of individuals is by means of a distance function that measures the disagreement between them, and this approach is followed in the paper. Thus, in particular, the construction of aggregation rules based on the minimization of distance functions inherits the normative appeal of consensus. Clearly, the distance-based approaches to aggregation theory are not limited to the construction of aggregation rules, but can fruitfully be applied to the comparison of aggregation procedures as well as to the geometric representation and to generalizations of the aggregation problems as discussed in the paper. A discussion of related problems such as the complexity of distance-based aggregation rules is also included.

G. Beliakov, T. Calvo and S. James ("On Penalty-Based Aggregation Functions and Consensus") consider the problem of aggregating individual preferences in order to arrive at a group consensus in contexts like elections where a candidate must be chosen that best represents the individuals' differing opinions, sport competitions and the fusion of sensor readings. In these applications the aggregated result should be as close as possible to the individual inputs giving rise to the need of methods that minimize this difference and this is what penalty-based aggregation functions are about drawing upon various notions of a "difference". One of more difficult issues when aggregating preferences is how to best assign numerical scores if only ordinal or pairwise preferences are given. In terms of penalty-based aggregation, the problem is further complicated by determining how the penalties should be calculated. The rationale behind using certain functions and certain measures of distance or penalty is context dependent. To use arithmetic means in group decision making, for example, implies a faith in the individuals to make accurate and sincere judgments that will not skew the results. Medians are not as susceptible in this way. When using aggregation methods, how consensus is interpreted will affect how it is achieved. A measure of distance or deviation from this value, or the imposition of a penalty for not having consensus has been studied in various forms, The authors draw upon the results on the penalty-based aggregation functions and penalty functions in general. They present some well used definitions of penalty and show how some aggregation functions correspond to minimizing the overall penalty associated with a given input vector. They consider some alternative frameworks of penalty and also introduce the idea of aggregating penalties using the OWA operator. It is shown that the penalty-based aggregation functions provide a natural framework for mathematical interpretations of consensus.

I. Contreras, M.A. Hinojosa and A.M. Mármol ("Ranking Alternatives in Group Decision-Making with Partial Information. A Stable Approach") propose in their paper some procedures for constructing global rankings of alternatives in situations in which each member of a group is able to provide imprecise or partial 
information on his/her preferences about the relative importance of criteria that have to be taken into account. The authors first propose an approach based on the assumption that the final evaluation depends on the complete group since no possibility exists that the group might split into coalitions that look for more favorable solutions for the [the] coalition members. To this end, the partial information on criteria weights provided by each individual is transformed into ordinal information on the alternatives, and then the aggregation of individual preferences is addressed within a distance-based framework. In a second approach, the possibility of coalition formation is considered, and the goal is to obtain rankings in which disagreements of all the coalitions are taken into account. These rankings will exhibit an additional property of collective stability in the sense that no coalition will have an incentive to abandon the group. This last approach may be of interest in political decisions where different sectors have to be incorporated into a joint evaluation process aiming at a consensus across all possible subgroups.

M. Brunelli, R. Fullér and J. Mezei ("Opinion Changing Aversion Functions for Group Settlement Modeling") consider opinion changing aversion (OCA) functions which are used to quantify the decision makers' resistance to opinion changing. The authors obtain a collective representation of preferences by solving a non-linear optimization problem to minimize the total level of disagreement and seeking an optimal, consensual, solution, the least disagreed one. Whenever such a consensual solution has to be found, a single valued, nonnegative cost function, an opinion changing aversion (OCA) function, is assigned to each decision maker and then the overall cost is minimized. The authors focus on the quadratic OCA functions and show that the group decision (or settlement) boils down to the center of gravity of the opinions of the decision makers. It is shown that if each expert has a quadratic opinion changing aversion function, then the minimum-cost solution is the weighted average of the individual optimal solutions where the weights are the relative importances of the decision makers. The authors consider the minimumcost solutions for group settlements under crisp and fuzzy budget constraints.

S. Montes, D. Martinetti, S. Díaz and S. Montes ("Statistical Preference as a Tool in Consensus Processes") deal with a so called statistical preference, a modern method of comparing probability distributions in the setting of consensus processes in which the intensities of preference can be expressed by means of probability distributions instead of single values. Since classical methods do not provide the possibility of comparing any pair of probability distributions, statistical preference is considered in the paper. One of its most remarkable advantages is that it allows to compare any pair of probability distributions. The authors study in depth some properties of this method and the relationship between the most commonly employed stochastic dominance and statistical preference. They also consider some of the most important families of distributions and analyze statistical preference among probability distributions in the same families.

Part II is concerned with issues underlying the meaning, composition and outcomes of individual and group decision making as well as social choice that are of relevance for consensus and consensual processes. It includes concepts and models dealing with social choice, group characterization and identification, veto power distribution, etc. 
M. Regenwetter and A. Popova ("Consensus with Oneself: Within-person Choice Aggregation in the Laboratory") follow their former efforts to [to] crossfertilize individual and social choice research[,] and apply behavioral social choice concepts to individual decision making. Though repeated individual choice among identical pairs of choice alternatives often fluctuates dramatically over even very short time periods, social choice theory usually ignores this because it identifies each individual with a single fixed weak order. Behavioral individual decision research may expose itself to Condorcet paradoxes because it often interprets a decision maker's modal choice (i.e., majority choice) over repeated trials as revealing their "true" preference. The authors investigate the variability in choice behavior within each individual in the research lab. Within that paradigm, they look for evidence of Condorcet cycles, as well as for the famed disagreement between the Condorcet and Borda aggregation methods. They also illustrate some methodological complexities involved with likelihood ratio tests for Condorcet cycles in paired comparison data.

D. Dimitrov ("The Social Choice Approach to Group Identification") gives an overview of selected topics from the theory of group identification intended to answer the question: given a group of individuals, how to define a subgroup in it? The problem of group identification is then viewed as a process of group formation. As a starting point the author uses different axiomatic characterizations of the "libera" rule for group identification whereby the group consist of those and only those individuals who view themselves as members of the group. The focus of the paper is then on consent rules and recursive procedures for collective determination in which the opinions of other individuals in the society also count. Finally, the author addresses recent developments in the literature with respect to gradual opinions and group identity functions.

A. Laruelle and F. Valenciano ("Consensus versus Dichotomous Voting") consider consensus [meant] as a general, maybe unanimous, agreement among possibly different views. Reaching a consensus is often a complex and difficult process involving adjustments, concessions, threats and bluffing, with no general rules, and dependent on the particular context. In which social rules, customs, past experience and communication constraints play a role. By contrast, dichotomous voting rules are in principle simple mechanisms for making decisions by using a vote to settle differences of view: the winning side enforces the decision to accept or reject the proposal on the table. Thus, these rules may be viewed in their spirit completely opposed to the idea of consensus. Nevertheless, it is often the case that a committee whose only formal mechanism to make decisions is a specified dichotomous voting rule reaches a consensus about an issue. Moreover, in many such cases the final vote is a purely formal act ratifying the agreement resulting from a consensual process and dichotomous voting rules which are a means of making decisions by using votes to settle differences of view. A natural question is therefore: How then can it often be the case that a committee whose only formal mechanism for decision-making is a dichotomous voting rule reaches a consensus? In this paper, based on a game-theoretic model developed in the authors' previous papers, an answer to this question is provided. 
J. Mercik ("On a priori Evaluation of Power of Veto") considers primarily the evaluation of power when some players have the right to veto, i.e. to stop the action of others permanently or temporarily. In certain cases, it is possible to calculate a value of power of veto attributed to the decision maker and to give the exact value of the power index as well. In other cases, it is only possible to compare the situation with and without veto attribute. In this paper the author analyzes the power of a player with a right to veto, expecting that the difference between the power of the player with veto and his or her power without veto makes it possible to evaluate directly or indirectly the power of veto itself.

Part III focuses on the environment and substantive content of consensus in various fields as well as provides an overview of approaches to measuring the degree of consensus, and some related topics.

H. Nurmi ("Settings of Consensual Processes: Candidates, Verdicts, Policies") considers the setting of social choice theory which basically deals with mutual compatibilities of various choice criteria or desiderata, and thus provides a natural angle to look at methods for finding consensus. The author distinguishes between three types of settings of consensus-reaching. Firstly, one may be looking for the correct decision. This is typically the setting where the participants have different degrees of expertise on an issue to be decided. Also jury decision making falls into this category. Secondly, the setting may involve the selection of one out of a set of candidates, for instance for a public office. Thirdly, one may be looking for a policy consensus. This setting is otherwise similar to the candidate choice setting, but usually involves more freedom in constructing new alternatives. The author first provides a review of these settings and relevant results in each one of them, and then discusses the implications of some choice paradoxes to consensus-reaching methods.

M. Martínez-Panero ("Consensus Perspectives: Glimpses into Theoretical Advances and Applications") gives a survey of polysemic meanings of consensus from several points of view, ranging from philosophical aspects and characterizations of several quantification measures within the social choice framework, paying also attention to aspects of judgment aggregation as well as fuzzy or linguistic approaches, to practical applications in decision making and biomathematics, to name a few. More specifically, the author first presents some philosophical aspects of consensus essentially focused on the doctrine that men are joined together within a society by a contract with explicit or hidden agreements, as Rousseau believed. Then, he outlines some further developments and connections, such as the link between Rousseau and Condorcet. The author also distinguishes between the concept of consent and the more technical and recent idea of consensus as appearing in modern political science and sociology. Next, he deals with several formal approaches to consensus mainly from the social choice framework, and some distance based, fuzzy or linguistic points of view. Moreover, he points out some aspects of an emergent research field focused on judgment aggregation, and concludes with a presentation of some applications as signs of the power of consensus-based methods in practice, a reference to the way of aggregating different estimates of each candidate through a median-based voting system. 
J. Alcalde-Unzu and M. Vorsatz ("Measuring Consensus: Concepts, Comparisons, and Properties") study approaches of how to measure the similarity of preferences in a group of individuals which is what they mean by consensus. First, the consensus for two individuals is determined and then the average over all possible pairs of individuals in the society is calculated. In the dual approach, first, the consensus between two alternatives is determined and then the average over all possible pairs of alternatives is calculated. The authors show that the choice between the two measures used in the above processes reduces to the choice between different monotonicity and independence conditions. Finally, some recent approaches are surveyed that take into account the fact that alternatives which are on the average ranked higher by the members of the society are more important for the social choice and should therefore be assigned a higher weight while calculating the consensus.

J.L. García-Lapresta and D. Pérez-Román ("Measuring Consensus in Weak Orders") consider the problem of how to measure consensus in groups of agents when they show their preferences over a fixed set of alternatives or candidates by means of weak orders (complete preorders). Consensus is here related to the degree of agreement in a committee, and agents do not need to change their preferences. The authors introduce a new class of consensus measures on weak orders based on distances, and analyze some of their properties paying special attention to seven well-known distances. They extend Bosch's consensus measure to the context of weak orders when indifference among different alternatives is allowed, and consider some additional properties like a maximum dissension (in each subset of two agents, the minimum consensus is only reached whenever preferences of agents are linear orders and each one is the inverse of the other), reciprocity (if all individual weak orders are reversed, then the consensus does not change) and homogeneity (if we replicate a subset of agents, then the consensus in that group does not change). Then, the authors introduce a class of consensus measures based on the distances among individual weak orders paying special attention to seven specific metrics: discrete, Manhattan, Euclidean, Chebyshev, cosine, Hellinger, and Kemeny.

L. Roselló, F. Prats, N. Agell and M. Sánchez (“A Qualitative Reasoning Approach to Measure Consensus") introduce a mathematical framework, based on the absolute order-of-magnitude qualitative model, which makes it possible to develop a methodology to assess consensus among different evaluators who use ordinal scales in group decision-making and evaluation processes. The concept of entropy is introduced in this context and the algebraic structure induced in the set of qualitative descriptions given by evaluators is studied. The authors prove that it is a weak partial semilattice structure which under some conditions takes the form of a distributive lattice. The definition of the entropy of a qualitatively-described system enables us, on the one hand, to measure the amount of information provided by each evaluator and, on the other hand, to consider a degree of consensus among the evaluation committee. The methodology presented makes it possible to manage situations when the assessment given by experts involves different levels of precision. In addition, when there is no consensus within the group decision, an automatic process measures the effort needed to reach consensus. 
M. Xia and Z. Xu ("On Consensus in Group Decision Making Based on Fuzzy Preference Relations") propose a method to derive the multiplicative consistent fuzzy preference relation from an inconsistent fuzzy preference relation. The fundamental characteristic of the method proposed is that it can get a consistent fuzzy preference relation taking into account all the original preference values without translation. Then, the authors develop an algorithm to transform a fuzzy preference relation into the one with the weak transitivity by using the original fuzzy preference relation and the constructed consistent one. After that, the authors propose an algorithm to help the decision makers reach an acceptable consensus in group decision making. It is worth pointing out that the group fuzzy preference relation derived by using the method proposed is also multiplicative consistent if all individual fuzzy preference relations are multiplicative consistent. The results obtained are illustrated by some examples.

S. Zadrożny, J. Kacprzyk and Z.W. Raś ("Supporting Consensus Reaching Processes under Fuzzy Preferences and a Fuzzy Majority via Linguistic Summaries and Action Rules") deal with the classic approach to the evaluation of the degree of consensus due to Kacprzyk and Fedrizzi $(1986,1988,1989)$ in which a soft degree of consensus has been introduced as a degree to which, for instance, "most of the important individuals agree as to almost all of the relevant options". The fuzzy majority is equated with a fuzzy linguistic quantifiers (most, almost all, ...) and handled via Zadeh's classic calculus of linguistically quantified propositions and Yager's OWA (ordered weighted average) operators. The consensus reaching process is run by a moderator who may need a support which is provided by a novel combination of: first, the use of the a soft degree of consensus due, and then the linguistic data summaries, in particular in its protoform based version proposed by Kacprzyk and Zadrożny to indicate in a natural language some interesting relations between individuals and options to help the moderator to identify crucial (pairs of) individuals and/options which pose some threats to the reaching of consensus. Third, using results obtained in the authors' recent paper, additionally a novel data mining tool, a so-called action rule proposed by Raś and Wieczorkowska is employed. The action rules are used in the context considered to find the best concessions to be offered to the individuals for changing their preferences to increase the degree of consensus.

Part IV includes contributions which deal with the implementation of theoretical models within decision support systems for running consensus reaching sessions, notably in the Web environment, and some more important application areas, including broadly perceived multicriteria decision making.

I.J. Pérez, F.J. Cabrerizo, M.J. Cobo, S. Alonso and E. Herrera-Viedma ("Consensual Processes Based on Mobile Technologies and Dynamic Information") present a prototype of a group decision support system based on mobile technologies and dynamic information. It is assumed that the users can run the system on their own mobile devices in order to provide their preferences anytime and anywhere. The system provides consensual and selection support to deal with dynamic decision making situations. Furthermore, the system incorporates a mechanism that makes it possible to manage dynamic decision situations in which some information about the problem is not constant throughout the time. It provides a more realistic decision 
making setting through high dimensional or dynamic set of alternatives, focussing the discussion on a subset of them that changes in each stage of the process. The experts' preferences are represented by using a linguistic approach. Therefore, the authors provide a new linguistic framework that is mobile and dynamic, to deal with group decision making problems.

L. Iandoli ("Building Consensus in On-line Distributed Decision Making: Interaction, Aggregation and Construction of Shared Knowledge") discusses the possibility of exploiting large-scale knowledge sharing and mass interaction taking place on the Internet to build decision support systems based on distributed collective intelligence. Pros and cons of currently available collaborative technologies are reviewed with respect to their ability to favor knowledge accumulation, filtering, aggregation and consensus formation. In particular, the author focuses on a special kind of collaborative technologies, a so called online collaborative mapping, whose characteristics can overcome some limitations of more popular collaborative tools, in particular thanks to their capacity to support collective sense-making and the construction of shared knowledge objects. The author discusses some contributions in the field and argues that the combination of online mapping and computational techniques for belief aggregation can provide an interesting basis to support the construction of systems for distributed decision-making.

F. Mata, J,.C. Martínez and R. Rodríguez ("A Web-based Consensus Support System Dealing with Heterogeneous Information") show a novel Web application of a consensus support system to carry out consensus reaching processes with heterogeneous information, i.e. the decision makers may use different information domains (in particular: numeric, interval-valued and linguistic assessments) to express their opinions. The software application developed has the following main characteristic features: it automates virtual consensus reaching processes in which experts may be put in different places, experts may use information domains near their work areas to provide their preferences and it is possible to run the system on any computer and operating system. This application may be seen as a practical development of a theoretical research on consensus modeling. It could be used by any organization to carry out virtual consensus reaching processes.

J. Ma, G.-G. Zhang and J. Lu (“A Fuzzy Hierarchical Multiple Criteria Group Decision Support System - Decider - and its Application") discuss Decider, a Fuzzy Hierarchical Multiple Criteria Group Decision Support System (FHMC-GDSS) designed for dealing with subjective, in particular linguistic, information and objective information simultaneously to support group decision making particularly focused on evaluation. The authors introduce first the fuzzy aggregation decision model, functions and structure of the Decider. The ideas of how to resolve decision making and evaluation problems encountered in the development and implementation of Decider are presented, and two real applications of the Decider system are briefly illustrated. Finally, some further future research in the area are briefly outlined.

D. Ben-Arieh and T. Easton ("Product Design Compromise Using Consensus Models") discuss the costs associated with decision making using group consensus, and then describe three methods of reaching a minimum cost consensus assuming quadratic costs for a single criterion decision problem. The first method finds the group opinion (consensus) that yields the minimum cost of reaching 
throughout the group. The second method finds the opinion with the minimum cost of the consensus providing that all experts must be within a given threshold of the group opinion. The last method finds the maximum number of experts that can fit within the consensus, given a specified budget constraint. In all of them the consensus process is defined as a dynamic and interactive group decision process, which is coordinated by a moderator, who helps the experts to gradually move their opinions until a consensus is reached. The work focuses on product design compromise and discusses how group consensus can be used in this process, and demonstrates the importance of the consensus process to the product design compromise process, and presents there models as mentioned above that can be used to obtain such a compromise.

We wish to thank all the contributors for their excellent work. All the contributions were anonymously peer reviewed by at least two reviewers, and we also wish to express our thanks to them. We hope that the volume will be interesting and useful to the entire research community working in diverse fields related to group decision making, social choice, consensual processes, multiagent systems, etc. as well as other communities in which people may find the presented tools and techniques useful to formulate and solve their specific problems.

We also wish to thank Dr. Tom Ditzinger and Mr. Holger Schaepe from Springer for their multifaceted support and encouragement.

November 2010

E. Herrera-Viedma

J.L. García-Lapresta

J. Kacprzyk

H. Nurmi

M. Fedrizzi

S. Zadrożny 


\section{Contents}

Part I: Basic Issues

Distance-Based Aggregation Theory .................. 3

Daniel Eckert, Christian Klamler

On Penalty-Based Aggregation Functions and Consensus ....

Gleb Beliakov, Tomasa Calvo, Simon James

Ranking Alternatives in Group Decision-Making with

Partial Information: A Stable Approach

I. Contreras, M.A. Hinojosa, A.M. Mármol

Opinion Changing Aversion Functions for Group

Settlement Modeling

Matteo Brunelli, Robert Fullér, József Mezei

Statistical Preference as a Tool in Consensus Processes ......

I. Montes, D. Martinetti, S. Díaz, S. Montes

Part II: Aspects of Group Decision Making, Social Choice and Voting

Consensus with Oneself: Within-Person Choice Aggregation

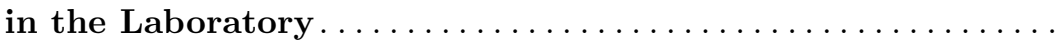

Michel Regenwetter, Anna Popova

The Social Choice Approach to Group Identification

Dinko Dimitrov

Consensus versus Dichotomous Voting

Annick Laruelle, Federico Valenciano 
On a Priori Evaluation of Power of Veto

Jacek Mercik

Part III: Various Aspects of Consensus, Its Measuring and Reaching

Settings of Consensual Processes: Candidates, Verdicts, Policies

Hannu Nurmi

Consensus Perspectives: Glimpses into Theoretical Advances and Applications

Miguel Martínez-Panero

Measuring Consensus: Concepts, Comparisons, and

Properties

Jorge Alcalde-Unzu, Marc Vorsatz

Measuring Consensus in Weak Orders

José Luis García-Lapresta, David Pérez-Román

A Qualitative Reasoning Approach to Measure Consensus . . 235

Llorenç Roselló, Francesc Prats, Núria Agell, Mónica Sánchez

On Consensus in Group Decision Making Based on Fuzzy Preference Relations.

Meimei Xia, Zeshui Xu

Supporting Consensus Reaching Processes under Fuzzy Preferences and a Fuzzy Majority via Linguistic Summaries and Action Rules 289

Stawomir Zadrożny, Janusz Kacprzyk, Zbigniew W. Raś

\section{Part IV: Modern Trends in Consensus Reaching Support, and Applications}

Consensual Processes Based on Mobile Technologies and

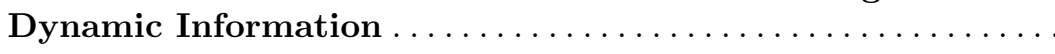

I.J. Pérez, F.J. Cabrerizo, M.J. Cobo, S. Alonso, E. Herrera-Viedma

Building Consensus in On-Line Distributed Decision Making: Interaction, Aggregation and the Construction of Shared Knowledge ....................... 339 Luca Iandoli 
A Web-Based Consensus Support System Dealing with

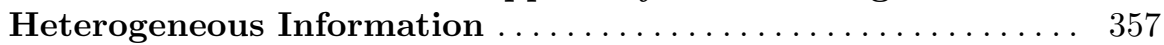
Francisco Mata, Juan Carlos Martínez, Rosa Rodríguez

A Fuzzy Hierarchical Multiple Criteria Group Decision Support System - Decider - and Its Applications ......... 383 Jun Ma, Guangquan Zhang, Jie Lu

Product Design Compromise Using Consensus Models ...... 405 David Ben-Arieh, Todd Easton

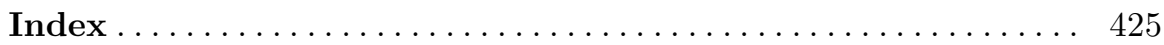

Author Index ................................ 427 